\title{
Population pharmacokinetics of orally administered mefloquine in healthy volunteers and patients with uncomplicated Plasmodium falciparum malaria
}

\author{
Stephanie E. Reuter ${ }^{1,2}$, Richard N. Upton ${ }^{1,2}$, Allan M. Evans ${ }^{1,2}$, Visweswaran Navaratnam ${ }^{3,4}$ and Piero L. Olliaro $5,6 *$ \\ ${ }^{1}$ School of Pharmacy \& Medical Sciences, University of South Australia, Adelaide, SA, Australia; ${ }^{2}$ Sansom Institute for Health Research, \\ University of South Australia, Adelaide, SA, Australia; ${ }^{3}$ Centre for Drug Research, Universiti Sains Malaysia, Penang, Malaysia; ${ }^{4}$ Postgraduate, \\ Research and Strategic Development, Taylor's University, Selangor, Malaysia; ${ }^{5}$ UNICEF/UNDP/World Bank/WHO Special Programme for \\ Research \& Training in Tropical Diseases (TDR), Geneva, Switzerland; ${ }^{6}$ Centre for Tropical Medicine and Vaccinology, \\ Nuffield Department of Medicine, University of Oxford, Churchill Hospital, Oxford, UK
}

*Corresponding author. UNICEF/UNDP/World Bank/WHO Special Programme for Research \& Training in Tropical Diseases (TDR), World Health Organization, Avenue Appia 20, CH-1211 Geneva 27, Switzerland. Tel: +41-22-791-3734; Fax: +41-22-791-4774; E-mail: olliarop@who.int

Received 12 May 2014; returned 9 June 2014; revised 8 August 2014; accepted 29 September 2014

\begin{abstract}
Background: The determination of dosing regimens for the treatment of malaria is largely empirical and thus a better understanding of the pharmacokinetic/pharmacodynamic properties of antimalarial agents is required to assess the adequacy of current treatment regimens and identify sources of suboptimal dosing that could select for drug-resistant parasites. Mefloquine is a widely used antimalarial, commonly given in combination with artesunate.
\end{abstract}

Patients and methods: Mefloquine pharmacokinetics was assessed in 24 healthy adults and 43 patients with Plasmodium falciparum malaria administered mefloquine in combination with artesunate. Population pharmacokinetic modelling was conducted using NONMEM.

Results: A two-compartment model with a single transit compartment and first-order elimination from the central compartment most adequately described mefloquine concentration-time data. The model incorporated population parameter variability for clearance $(C L / F)$, central volume of distribution $\left(V_{C} / F\right)$ and absorption rate constant $\left(K_{\mathrm{A}}\right)$ and identified, in addition to body weight, malaria infection as a covariate for $V_{C} / F$ (but not $C L / F$ ). Monte Carlo simulations predict that falciparum malaria infection is associated with a shorter elimination half-life (407 versus $566 \mathrm{~h}$ ) and $T_{>\text {MIC }}$ (766 versus $893 \mathrm{~h}$ ).

Conclusions: This is the first known population pharmacokinetic study to show falciparum malaria to influence mefloquine disposition. Protein binding, anaemia and other factors may contribute to differences between healthy individuals and patients. As $V_{C} / F$ is related to the earlier portion of the concentration-time profiles, which occurs during acute malaria, and $\mathrm{CL} / \mathrm{F}$ is more related to the terminal phase during convalescence after treatment, this may explain why malaria was found to be a covariate for $V_{C} / F$ but not $C L / F$.

Keywords: PK, pharmacometrics, P. falciparum

\section{Introduction}

Malaria is a leading cause of mortality and morbidity in developing areas of the world, with an estimated 216 million individuals infected each year, of which $80 \%$ are within sub-Saharan Africa and South America. ${ }^{1}$ The current WHO recommendation for the treatment of uncomplicated Plasmodium falciparum malaria is with artemisinin combination therapy comprising two schizontocidal drugs with independent modes of action, specifically a rapid-acting artemisinin-derived compound administered with a more slowly eliminated antimalarial (e.g. mefloquine). ${ }^{2}$ Artesunate and mefloquine combination treatments have been shown to achieve consistently high efficacy rates and reduced malaria morbidity, including in areas of multidrug resistance. ${ }^{3}$

Mefloquine is an orally administered blood schizontocide, active against the erythrocytic stages of $P$. falciparum infection. ${ }^{4}$ The total recommended dose of mefloquine, administered in combination with artesunate for the treatment of malaria infection, is $25 \mathrm{mg} / \mathrm{kg}$ typically given as a split dose over 2 days $(15+10 \mathrm{mg} / \mathrm{kg})$ or $24 \mathrm{mg} / \mathrm{kg}$ administered over 3 days $(8+8+8 \mathrm{mg} / \mathrm{kg}) .^{4-7}$ Whilst this 
dose recommendation has been established as efficacious in the treatment of uncomplicated falciparum malaria in adult patients, the dose regimen is largely empirical and a better understanding of the pharmacokinetic and pharmacodynamic properties of mefloquine is required to ensure rational use and optimal dosing regimens.

Mefloquine pharmacokinetics is characterized by low apparent oral clearance, a large apparent volume of distribution and consequently a long terminal half-life; however, considerable interindividual differences have been reported. ${ }^{4}$ Given that treatment efficacy is dependent on maintenance of parasiticidal blood levels until all parasites are eliminated from the body, this slow elimination phase is an important determinant of therapeutic outcomes. ${ }^{6,8}$ Research has suggested that mefloquine disposition in patients with falciparum malaria is different from that in healthy volunteers, with a substantially reduced half-life ( $\sim 2$ weeks compared with $\sim 3$ weeks). ${ }^{4,9-12}$ Given that traditional (separate) pharmacokinetic studies in relatively small subsets of participants are, for the most part, underpowered to ascertain the factors that contribute to the variability in pharmacokinetics, it is not known whether these observed differences between patients and healthy volunteers are due to the presence of malaria infection or could be accounted for by differences in other factors such as body weight, gender, etc. In order to investigate this further, a population pharmacokinetic approach was used to provide estimates of mefloquine pharmacokinetic parameters and an assessment of the sources of variance in pharmacokinetic parameters in patients with uncomplicated $P$. falciparum malaria and healthy volunteers.

\section{Patients and methods}

Pharmacokinetic data were obtained from two clinical studies conducted in healthy volunteers and patients with uncomplicated $P$. falciparum malaria. ${ }^{12,13}$ Non-compartmental pharmacokinetic analysis of the concentration-time data from these studies has previously been described. ${ }^{12}$

The clinical studies were conducted at the Hospital for Tropical Disease, Mahidol University (Bangkok, Thailand) and were reviewed and approved by the institutional Human Research Ethics Committee. Participants were fully informed of the study procedures and provided written informed consent prior to study initiation. The studies were conducted in accordance with the study protocols, the Declaration of Helsinki and the principles of Good Clinical Practice.

\section{Study design}

The pharmacokinetics of mefloquine, after administration of one of two treatment formulations, were to be assessed in 24 healthy adult male and non-pregnant female volunteers (healthy volunteer study) and 50 male and non-pregnant female patients with slide-proven uncomplicated $P$. falciparum malaria (patient study).

In the clinical studies, mefloquine was administered in combination with artesunate as either a coformulated product (fixed formulation) or as separate products (non-fixed formulation). The coformulated product was administered as tablets containing $200 \mathrm{mg}$ of mefloquine and $100 \mathrm{mg}$ of artesunate (FarManguinhos, Rio de Janeiro, Brazil; Batches $\# 070008$ and \#069002). The separate products comprised mefloquine administered as $250 \mathrm{mg}$ tablets (Roche, Basel, Switzerland; Batch \#B1100) and $50 \mathrm{mg}$ artesunate tablets (Guilin Pharmaceutical, Guangxi, China; Batch \#031201).

The healthy volunteer study was conducted according to a randomized, crossover design with a 90 day washout period between treatment periods. Healthy volunteers were administered a single oral dose of
$400 \mathrm{mg}$ of mefloquine as the coformulated product or $500 \mathrm{mg}$ of mefloquine as a separate product. In both study periods, blood samples were collected for analysis of plasma mefloquine concentrations prior to dosing (0 h) and at $0.25,0.5,0.75,1,1.5,2,3,4,6,8,12,24,48,72,120,168,336$, $504,672,1008,1344,1680$ and $2160 \mathrm{~h}$ after treatment administration.

The patient study was conducted according to a randomized, parallel design with patients randomly allocated to receive multiple dosing with one of the two treatment formulations according to a $1: 1$ ratio. Patients received either $400 \mathrm{mg}$ of mefloquine as the coformulated product each day for 3 days, or $25 \mathrm{mg} / \mathrm{kg}$ mefloquine administered as a separate product as a spilt dose over 2 days ( $15 \mathrm{mg} / \mathrm{kg}+10 \mathrm{mg} / \mathrm{kg}$ ). For patients administered the coformulated product, blood samples were collected prior to dosing ( $0 \mathrm{~h}$ ) and at $0.25,0.5,0.75,1,1.5,2,3,4,6,8,12,24$ (pre-dose \#2), 48 (pre-dose \#3), 72, 120, 168, 336, 504 and $672 \mathrm{~h}$ after administration of the first dose. For patients administered the separate products, blood samples were collected prior to dosing ( $0 \mathrm{~h}$ ) and at 24 (pre-dose \#2), 48, 96, $144,312,480$ and $648 \mathrm{~h}$ after administration of the first dose.

Schematic representations of the study designs are illustrated in Figure 1.

\section{Biological sample analysis}

Plasma samples were analysed for mefloquine concentrations by Universiti Sains Malaysia (Penang, Malaysia) using validated HPLC-UV methodology. ${ }^{12,14}$ The lower limit of quantification of mefloquine was $20 \mathrm{mg} / \mathrm{mL}$, with an accuracy of $98.9 \%$ and a coefficient of variation of $8 \%$.

Data for samples returning concentrations below the lower limit of quantification of the assay were not made available by the analytical laboratory. These samples and samples for which no values were reported (i.e. missing) were excluded from the pharmacokinetic dataset.

\section{Population pharmacokinetics}

Population pharmacokinetic modelling was conducted using NONMEM ${ }^{\circledR}$ VI (ICON Development Solutions, Ellicott City, MD, USA) software with a G77 complier and Wings for NONMEM interface (http://wfn.sourceforge.net). Full details of the model development protocol and model diagnostics are available as Supplementary data at JAC Online (http://jac.oxfordjournals. org/); the pharmacometric methods are briefly described below.

One-, two- and three-compartment models with first-order absorption and elimination from the central compartment were fitted to mefloquine plasma concentration-time data. Models with absorption lag time were screened to account for any time delay between administration and the beginning of drug absorption. In addition, more complex absorption models based on a chain of absorption transit compartments were also investigated. ${ }^{15}$ The pharmacokinetic models were parameterized (where appropriate) as clearance (CL/F), volume of distribution of the central compartment $\left(V_{C} / F\right)$, intercompartmental clearance $\left(C_{D} / F\right)$, volume of distribution of the peripheral compartment $(s)\left(V_{P} / F\right)$, absorption rate constant $\left(K_{A}\right)$ and absorption lag time. The model incorporated population parameter variability (comprising between-subject variability and between-occasion variability) and residual unexplained variability (comprising proportional and additive error).

Once the base structural model had been determined, the contributions of continuous (age, body weight and baseline parasite count) and categorical (gender, treatment formulation and presence of malaria infection) covariates to the population parameter variability were assessed using a forward selection-backward elimination procedure.

Model selection was based on the objective function value (minus twice the log-likelihood of the data) as well as visual inspection of the standard diagnostic plots. A statistically significant $(P<0.05)$ improvement in the comparison of nested models was defined as a decrease in the objective function value of $3.84 \mathrm{U}$ (for 1 degree of freedom). The final population pharmacokinetic model was evaluated through visual predictive checks and bootstrap analysis. 
(a)

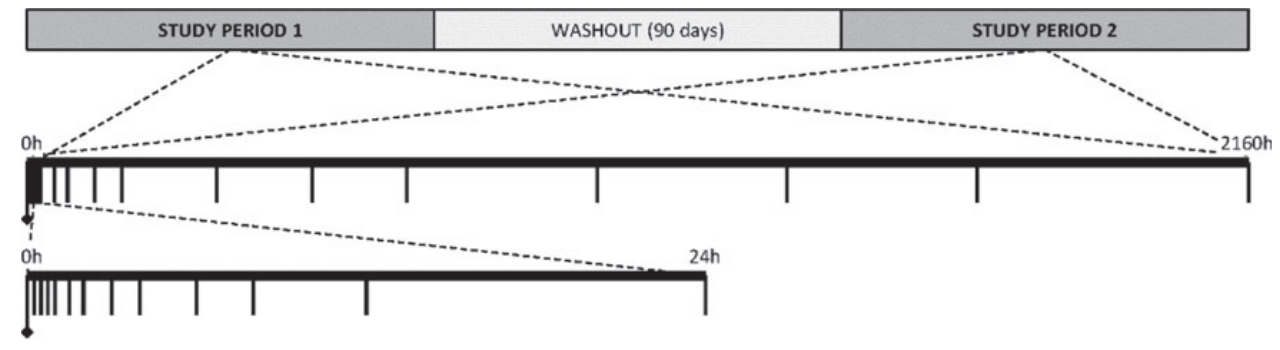

(b)

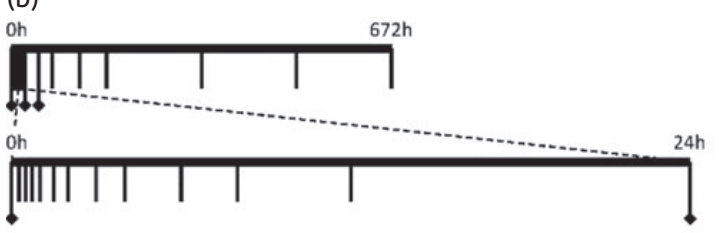

(c)

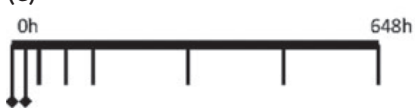

Figure 1. Schematic representation of clinical trial design for each study component. Dose administration time(s) are illustrated by black diamonds and blood sampling timepoints are indicated by vertical lines. (a) Healthy volunteer study: randomized, crossover study design with administration of a single dose of mefloquine (in combination with artesunate) in each study period (separate product in period 1 and then coformulated product in period 2 or vice versa) with intensive blood sampling over the first $24 \mathrm{~h}$ followed by longitudinal sampling until $2160 \mathrm{~h}$. (b) Patient study (coformulated product cohort): randomized, parallel study design with administration of daily mefloquine (in combination with artesunate) for 3 days with intensive blood sampling over the first $24 \mathrm{~h}$ followed by longitudinal sampling until $672 \mathrm{~h}$. (c) Patient study (separate product cohort): randomized, parallel study design with administration of daily mefloquine (in combination with artesunate) for 2 days with longitudinal blood sampling until $648 \mathrm{~h}$.

Table 1. Key study characteristics

\begin{tabular}{|c|c|c|c|c|c|}
\hline Population & Study treatment & Mefloquine dose & Regimen & $\begin{array}{l}\text { Number of } \\
\text { subjects }\end{array}$ & $\begin{array}{c}\text { Number of } \\
\text { pharmacokinetic } \\
\text { samples }\end{array}$ \\
\hline Healthy subjects & $\begin{array}{l}\text { fixed formulation } \\
\text { non-fixed formulation }\end{array}$ & $\begin{array}{l}400 \mathrm{mg} \\
500 \mathrm{mg}\end{array}$ & $\begin{array}{l}\text { single dose } \\
\text { single dose }\end{array}$ & 24 & $\begin{array}{l}468 \\
508\end{array}$ \\
\hline Malaria patients & $\begin{array}{l}\text { fixed formulation } \\
\text { non-fixed formulation }\end{array}$ & $\begin{array}{l}1200 \mathrm{mg} \\
25 \mathrm{mg} / \mathrm{kg}\end{array}$ & $\begin{array}{l}\text { split dose over } 3 \text { days }(400+400+400 \mathrm{mg}) \\
\text { split dose over } 2 \text { days }(15+10 \mathrm{mg} / \mathrm{kg})\end{array}$ & $\begin{array}{l}20 \\
23\end{array}$ & $\begin{array}{l}337 \\
157\end{array}$ \\
\hline Total & & & & 67 & 1470 \\
\hline
\end{tabular}

\section{Monte Carlo simulation}

Given the differences in treatment regimens in the healthy volunteer and patient studies, the original data did not allow for direct comparison of the time course of mefloquine between the two populations. To make this comparison, the final population pharmacokinetic model was used to simulate datasets for 1200 healthy volunteers and 1075 patients with uncomplicated falciparum malaria (i.e. 25 complete datasets) after administration of $8 \mathrm{mg} / \mathrm{kg} /$ day mefloquine for 3 days. Clinically relevant parameters, half-life and $T_{>M I C}\left(\mathrm{MIC}=0.5 \mathrm{mg} / \mathrm{L}^{6}\right)$, were then determined for each population.

\section{Results}

Overall, 1470 plasma mefloquine concentration-time data points from 24 healthy volunteers and 43 patients with $P$. falciparum malaria were included in the population pharmacokinetic analysis. An additional 148 post-dose data points were excluded from the dataset as concentrations were less than the limit of quantification (107 samples for healthy volunteers and 41 samples for patients with malaria). A summary of the key study characteristics is presented in Table 1 and population characteristics (covariates) are summarized in Table 2.

A two-compartment model with a single transit compartment and first-order elimination from the central compartment was found to most adequately describe the mefloquine concentration-time data. The model incorporated population parameter variability for $C L / F, V_{C} / F$ and $K_{A}$; negligible variability was estimated for $\mathrm{CL}_{D} / \mathrm{F}$ and $V_{P} / F$ and these were therefore fixed-effect parameters. The population parameter variability for $\mathrm{CL} / \mathrm{F}$ and $V_{C} / F$ comprised between-occasion variability and between-subject variability, 
Table 2. Study population characteristics

\begin{tabular}{|c|c|c|c|}
\hline \multirow[b]{2}{*}{ Covariate } & \multirow[b]{2}{*}{ Healthy subjects } & \multicolumn{2}{|c|}{ Malaria patients } \\
\hline & & fixed formulation & non-fixed formulation \\
\hline Number of subjects & 24 & 20 & 23 \\
\hline Age (years), mean \pm SD (range) & $35.0 \pm 7.17(20.9-47.8)$ & $27.8 \pm 10.5(18.0-50.3)$ & $28.9 \pm 10.4(16.4-45.5)$ \\
\hline Body weight $(\mathrm{kg})$, mean $\pm S D$ (range) & $55.9 \pm 10.4(41.0-87.0)$ & $50.4 \pm 6.42(40.0-62.0)$ & $50.7 \pm 6.49(40.0-65.0)$ \\
\hline Height $(\mathrm{cm})$, mean \pm SD (range) & $158 \pm 7.98(145-180)$ & $161 \pm 5.89(150-170)$ & $160 \pm 7.31(147-174)$ \\
\hline Gender & 4 male/20 female & 17 male/4 female & 21 male/3 female \\
\hline Treatment formulation & 24 fixed/24 non-fixed & 20 fixed/0 non-fixed & 0 fixed/23 non-fixed \\
\hline Presence of malaria infection & 24 healthy/0 malaria & 0 healthy/20 malaria & 0 healthy/23 malaria \\
\hline Baseline parasite count (number/ $\mu \mathrm{L}$ ), mean \pm SD (range) & 0 & $28364 \pm 41663(69-145860)$ & $29330 \pm 45450(20-140280)$ \\
\hline
\end{tabular}
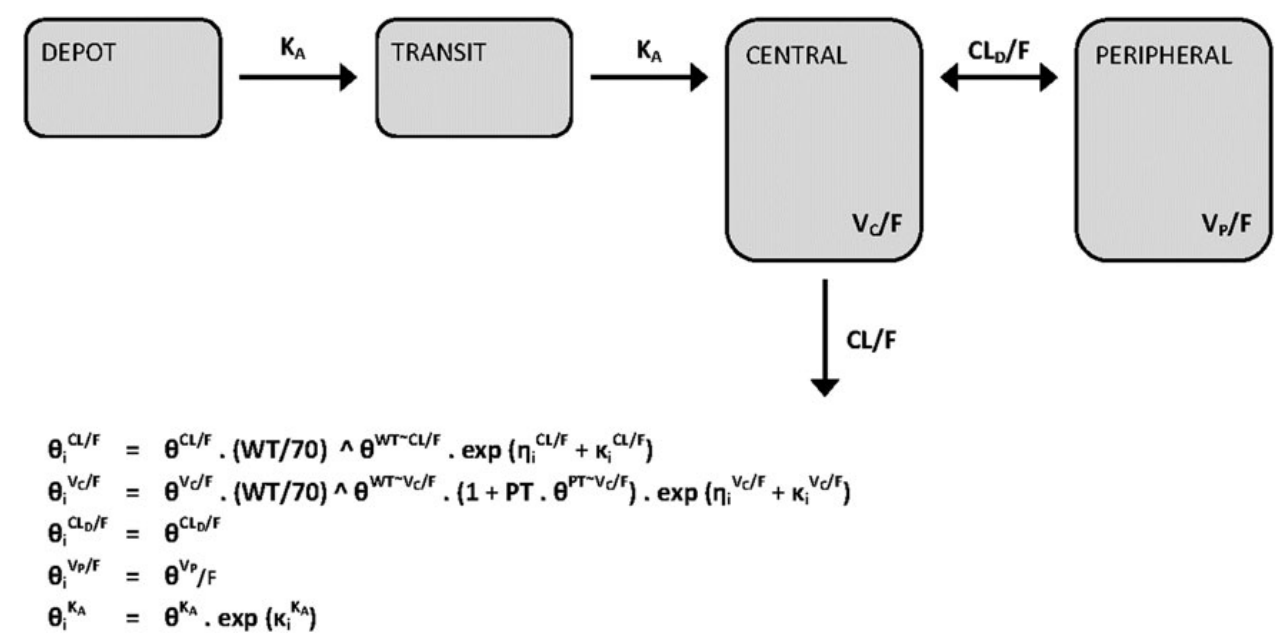

Figure 2. Schematic of the final population model describing the pharmacokinetics of mefloquine. $\theta_{i}$, individual parameter; $\theta$, population parameter; $\eta_{\mathrm{i}}$, random effect of between-subject variability; $\kappa_{i}$, random effect of between-occasion variability; $C L / F$, clearance; $V_{C} / F$, volume of distribution of the central compartment; $C L_{D} / F$, intercompartmental clearance; $V_{P} / F$, volume of distribution of the peripheral compartment; $K_{A}$, absorption rate constant; WT, body weight; PT, presence of malaria (where no malaria $=0$ and malaria infection $=1$ ); WT $\sim C L / F$, effect of weight on clearance; $W T \sim V_{C} / F$, effect of weight on central volume of distribution; $\mathrm{PT} \sim V_{C} / F$, effect of the presence of malaria on central volume of distribution.

whereas the model could only account for between-occasion variability for $K_{\mathrm{A}}$ with negligible between-subject variability. Combined proportional and additive residual unexplained variability was supported by the model.

Examination of the association between estimates of pharmacokinetic parameters generated from the structural model and covariates detected weight and the presence of malaria infection as potential covariates for $C L / F$ and $V_{C} / F$. Introduction of covariates into the structural model identified an effect of weight on $\mathrm{CL} / \mathrm{F}$ and weight and the presence of malaria infection on $V_{C} / F$. The inclusion of covariates substantially improved the model, in particular the inclusion of the presence of malaria as a covariate for $V_{C} / F$, which resulted in a reduction in the variability in this parameter and a 34 point reduction in the objective function value.

The population parameter estimates were $\mathrm{CL} / \mathrm{F}=1.22 \mathrm{~L} / \mathrm{h}$, $V_{C} / F=677 \mathrm{~L}, \mathrm{CL}_{D} / \mathrm{F}=1.34 \mathrm{~L} / \mathrm{h}, V_{\mathrm{P}} / \mathrm{F}=186 \mathrm{~L}$ and $K_{\mathrm{A}}=0.985 \mathrm{~h}^{-1}$, with the presence of malaria infection associated with a $40 \%$ reduction in $V_{C} / F$. The final population pharmacokinetic model is illustrated in Figure 2 and, based on the model diagnostics, was found to well characterize the mefloquine concentration-time data. Population parameter estimates of the final pharmacokinetic model are presented in Table 3. Median and 90\% prediction intervals obtained from 500 replicate datasets (bootstrap procedure) were comparable to parameter estimates from the original dataset, indicating the robustness and precision of the final population pharmacokinetic model (Table 3).

The visual predictive check demonstrated agreement between observed and simulated data, with the median and $90 \%$ prediction intervals of mefloquine concentrations closely predicted over the time course of the study for healthy volunteers administered $400 \mathrm{mg}$ of mefloquine as a single dose and patients with malaria administered $1200 \mathrm{mg}$ of mefloquine as split doses over 2 or 3 days (Figure 3 ).

Full details of the model performance are available as Supplementary data at JAC Online.

Output from the simulation of 1200 healthy subjects and 1075 malaria patients administered $8 \mathrm{mg} / \mathrm{kg} /$ day mefloquine for 3 days is presented in Figure 4 and the determined pharmacokinetic 
Reuter et al.

Table 3. Final model population parameter estimates of mefloquine pharmacokinetics and evaluation using bootstrap procedure (500 replicate datasets)

\begin{tabular}{|c|c|c|c|c|c|}
\hline Parameter & Final model: estimate & Bootstrap: median & Bootstrap: \%RSE & Shrinkage & Eta Bar $P$ \\
\hline \multicolumn{6}{|l|}{ Fixed effects } \\
\hline $\mathrm{CL} / \mathrm{F}(\mathrm{L} / \mathrm{h})$ & 1.22 & 1.22 & 10.4 & & \\
\hline$V_{C} / F(L)$ & 677 & 676 & 7.86 & & \\
\hline $\mathrm{CL}_{D} / \mathrm{F}(\mathrm{L} / \mathrm{h})$ & 1.34 & 1.33 & 14.4 & & \\
\hline$V_{\mathrm{P}} / \mathrm{F}(\mathrm{L})$ & 186 & 189 & 7.46 & & \\
\hline$K_{\mathrm{A}}\left(\mathrm{h}^{-1}\right)$ & 0.985 & 0.990 & 8.00 & & \\
\hline $\mathrm{WT} \sim \mathrm{CL} / \mathrm{F}$ & 0.405 & 0.424 & 67.6 & & \\
\hline$W T \sim V_{C} / F$ & 0.828 & 0.820 & 32.7 & & \\
\hline $\mathrm{PT} \sim V_{C} / \mathrm{F}$ & -0.400 & -0.403 & 14.7 & & \\
\hline \multicolumn{6}{|l|}{ Random effects } \\
\hline BSV CL/F (\%CV) & 28.5 & 26.0 & 38.2 & $20.2 \%$ & 0.818 \\
\hline BOV CL/F (\%CV) & 21.4 & 22.2 & 30.5 & $36.6 \% / 62.4 \%$ & $0.0982 / 0.0153$ \\
\hline BSV $V_{C} / F(\% C V)$ & 1.85 & 1.96 & 274 & $98.2 \%$ & 0.952 \\
\hline BOV $V_{C} / F(\% C V)$ & 36.5 & 35.8 & 10.2 & $0.190 \% / 53.5 \%$ & $0.322 / 0.0237$ \\
\hline BOV $K_{A}(\% C V)$ & 60.2 & 59.7 & 15.1 & $10.5 \% / 58.2 \%$ & 0.483/0.139 \\
\hline \multicolumn{6}{|l|}{ Residual variability } \\
\hline proportional (\%CV) & 22.7 & 22.9 & 4.94 & $7.69 \%$ & \\
\hline additive $(\mu \mathrm{g} / \mathrm{L})$ & 24.2 & 23.7 & 21.8 & $7.69 \%$ & \\
\hline
\end{tabular}

$\mathrm{CL} / \mathrm{F}$, clearance; $V_{C} / F$, volume of distribution of the central compartment; $C_{D} / F$, intercompartmental clearance; $V_{P} / F$, volume of distribution of the peripheral compartment; $K_{\mathrm{A}}$, absorption rate constant; WT CL/F, effect of weight on clearance; WT $V_{C} / F$, effect of weight on central volume of distribution; PT $\sim V_{C} / F$, effect of the presence of malaria infection on central volume of distribution; BSV, between-subject variability; $\mathrm{BOV}$, between-occasion variability; \%RSE, relative standard error; \%CV, coefficient of variation.

Refer to Figure 2 for structural representation of the final population pharmacokinetic model.

parameters are presented in Table 4. Results indicate that patients with uncomplicated $P$. falciparum malaria exhibit a $28 \%$ shorter elimination half-life (407 h compared with $566 \mathrm{~h}$ ) and a $14 \%$ reduction in $T_{>\text {MIC }}$ (766 h compared with $893 \mathrm{~h}$ ) compared with healthy controls. Very similar results were obtained when simulating the separate product treatment regimen $(15 \mathrm{mg} / \mathrm{kg}$ on day $1+10 \mathrm{mg} / \mathrm{kg}$ on day 2 ) (data not shown).

\section{Discussion}

The therapeutic effectiveness of long-acting antimalarials such as mefloquine is dependent on the maintenance of drug levels above the MIC until all parasites have been eliminated from the body (with the contribution of the host's immune system). ${ }^{6,8}$ Given that underdosing would have significant implications for treatment failure and may contribute to the selection of drug-resistant parasites, whereas overdosing could produce toxic side effects, additional research is required to establish the appropriateness of current dosing regimens.

The long elimination half-life of mefloquine is critical for treatment efficacy. ${ }^{6,8}$ Previous research has noted considerable interindividual variability in this parameter; $;^{4,9-12}$ however, the factors contributing to this variability have not been determined. Noncompartmental pharmacokinetic analysis of the data utilized in this study indicated a substantially lower elimination half-life of mefloquine in patients with uncomplicated $P$. falciparum malaria compared with healthy adult volunteers ( $294 \mathrm{~h}$ compared with $492 \mathrm{~h}$, respectively). ${ }^{12}$ For the first time, to our knowledge, the factors contributing to the variability in mefloquine pharmacokinetics between individuals have been investigated using a population pharmacokinetic approach.

In this study, a two-compartment model with a single transit compartment and first-order elimination from the central compartment best characterized the mefloquine concentration-time data. Previously, limited population pharmacokinetic analyses of mefloquine have been conducted and, for the most part, these analyses utilized one-compartment models due to sparse concentration-time data. ${ }^{6,16,17}$ On the other hand, Charles et al. ${ }^{18}$ recently described two-compartment mefloquine population pharmacokinetics in 1111 healthy military personnel administered mefloquine for malaria prophylaxis. Similarly, Svensson et al. ${ }^{19}$ described a two-compartment population pharmacokinetic model of mefloquine enantiomers in patients with falciparum malaria. Whilst there has been considerable variability in pharmacokinetic values reported from both population and traditional pharmacokinetic analyses, the population estimates of apparent clearance and total volume of distribution reported in the present study (1.22 L/h and $863 \mathrm{~L}$, respectively) are consistent with the low systemic clearance and large volume of distribution established for mefloquine. ${ }^{4}$

Not surprisingly, weight was identified as a significant covariate for both the elimination and distribution of mefloquine. This is in keeping with previously reported population pharmacokinetic models that identified body weight as an important factor for clearance $^{16,17}$ and volume of distribution. ${ }^{17,19}$ However, for the first time, this study was also able to demonstrate the 

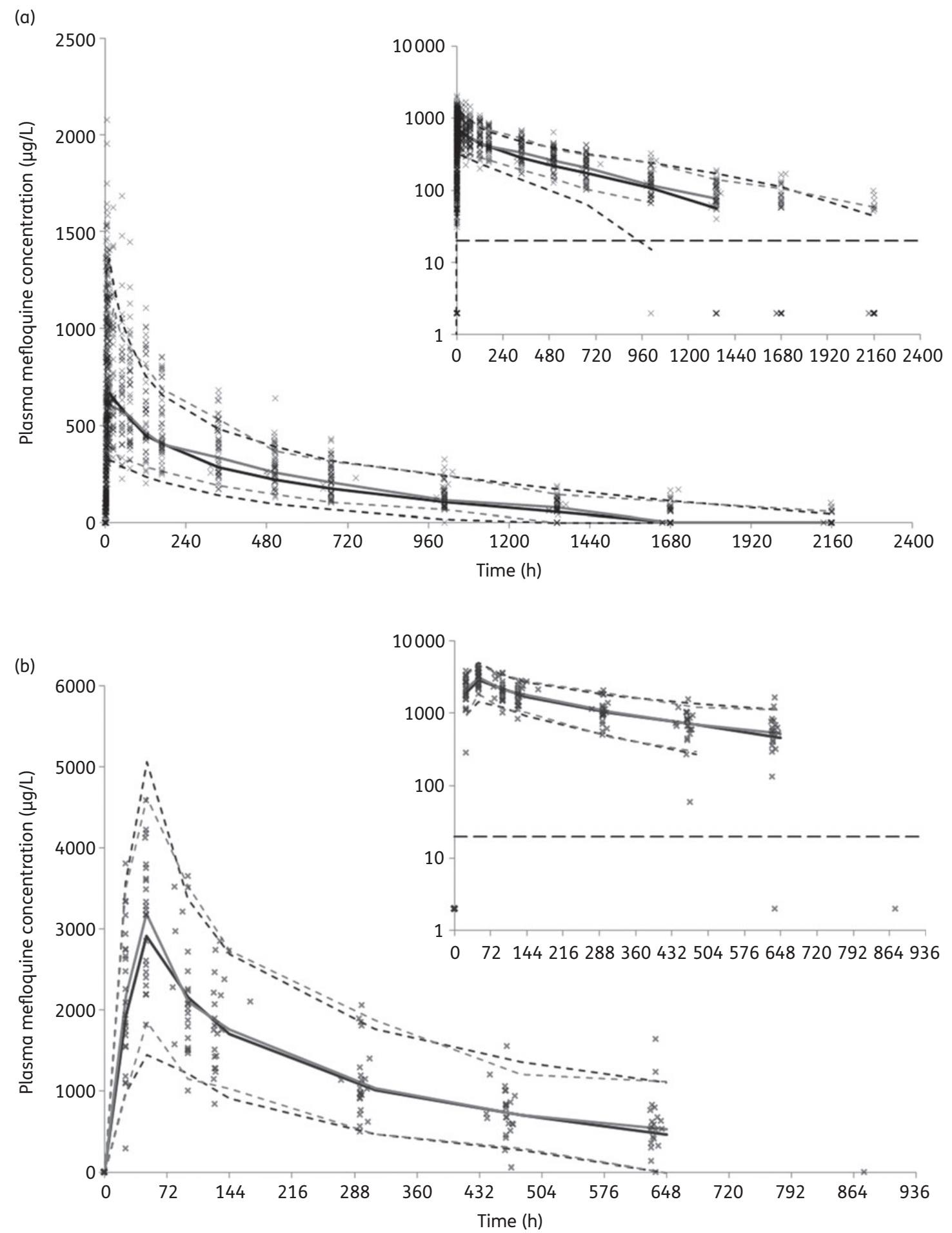

Figure 3. Visual predictive check of the median (continuous lines) and $90 \%$ prediction intervals (broken lines) for observed (grey) and simulated (black) data with individual observed data (crosses). (a) Plasma concentration-time data for healthy volunteers administered a single oral dose of $400 \mathrm{mg}$ of mefloquine. (b) Plasma concentration-time data for patients with uncomplicated $P$. falciparum malaria administered 1200 mg of mefloquine as a split dose over 2 days $(720+480 \mathrm{mg}$ ). (c) Plasma concentration-time data for patients with uncomplicated $P$. falciparum malaria administered 1200 mg of mefloquine as a split dose over 3 days $(400+400+400 \mathrm{mg})$. For illustrative purposes, individual observed concentration data below the limit of quantification of the assay have been designated a value of $2 \mu \mathrm{g} / \mathrm{L}$ in the graphical representation of data. The lower limit of quantification of the assay $(20 \mu \mathrm{g} / \mathrm{L})$ is illustrated by the horizontal broken line. 


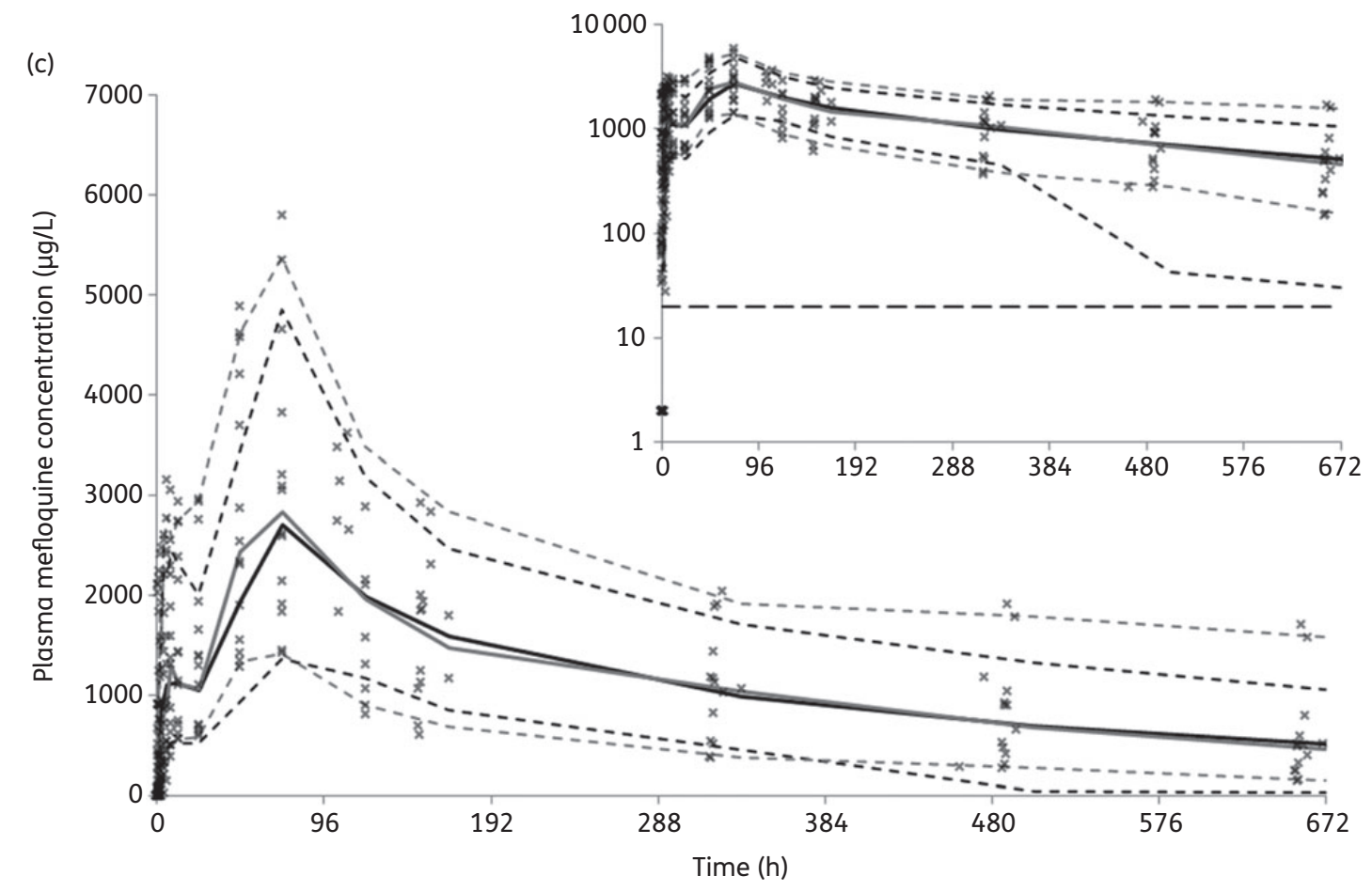

Figure 3. Continued

independent effect of the presence of malaria infection on mefloquine disposition, with a $40 \%$ lower central volume of distribution in patients compared with healthy adult subjects. Interestingly, while there have been no previous population studies examining mefloquine pharmacokinetics in both malaria patients and healthy adults, analyses conducted by Ashley et al. ${ }^{16}$ and Simpson et al. ${ }^{6}$ reported body temperature as a significant covariate for volume of distribution (with increasing temperature associated with reduced volume). Body temperature could conceivably be seen as a proxy for the presence of malaria infection, thereby supporting our findings, although parasitaemia seems to be a better marker of infection severity.

Results of the simulation of datasets for patients with malaria and healthy adults administered $8 \mathrm{mg} / \mathrm{kg} / \mathrm{day}$ mefloquine for 3 days indicated that the presence of malaria infection is associated with a substantially lower half-life and a reduction in $T_{>\text {MIC. }}$ Given that treatment effectiveness is characterized by the maintenance of blood mefloquine concentrations above the MIC for an adequate period of time to ensure maximum parasiticidal activity, this shorter elimination phase and duration of action could have implications for treatment outcomes. It should also be noted that the MIC may vary with location and time and calculations should be adapted accordingly.

The physiological mechanism accounting for the differences in mefloquine pharmacokinetics between malaria patients and healthy adults is unknown. One possible explanation may be due to differences in the plasma protein binding of mefloquine. It has previously been established that mefloquine extensively binds to plasma proteins, in particular acute-phase reactants such as $\alpha_{1}$-acid glycoprotein $\left(K_{A} \approx 10^{6} \mathrm{M}^{-1}\right) .{ }^{20}$ Furthermore, $\alpha_{1}$-acid glycoprotein levels have been shown to vary considerably in certain diseases, including malaria with a reported $168 \%$ increase in levels compared with healthy controls. ${ }^{21-23}$ This substantial increase in $\alpha_{1}$-acid glycoprotein levels in malaria would theoretically result in increased mefloquine plasma protein binding and thus a reduction in the apparent volume of distribution. Given that plasma $\alpha_{1}$-acid glycoprotein levels have also been shown to vary in pregnancy, infancy and HIV infection as well as states of malnutrition, ${ }^{21,22}$ the proposed relationship between mefloquine pharmacokinetics and $\alpha_{1}$-acid glycoprotein is likely to be an important factor in the determination of appropriate treatment guidelines for these patients. Furthermore, as race/ethnicity have also been shown to influence $\alpha_{1}$-acid glycoprotein levels, ${ }^{24}$ this may account, at least in part, for the considerable variability in mefloquine pharmacokinetics reported in the literature. However, it should be noted that changes in protein binding would also be expected to result in altered mefloquine clearance, a result that was not observed in this study. Given that, for drugs with a low hepatic extraction ratio, drug clearance is dependent on both the fraction unbound and intrinsic clearance, it is conceivable that the proposed increase in mefloquine protein binding in patients with malaria may also be accompanied by an increase in the intrinsic clearance of mefloquine, therefore resulting in no observed changes in mefloquine clearance. An alternative explanation is discussed below.

An important factor, which has not been investigated as part of this study, is the chirality of mefloquine. Mefloquine is administered clinically as a racemic mixture of two enantiomers, (-)-mefloquine and (+)-mefloquine, and therefore the stereoselective aspects of the disposition and effects of mefloquine should be considered. Previous research has demonstrated that the (-)-enantiomer was associated with higher apparent volume of distribution and 


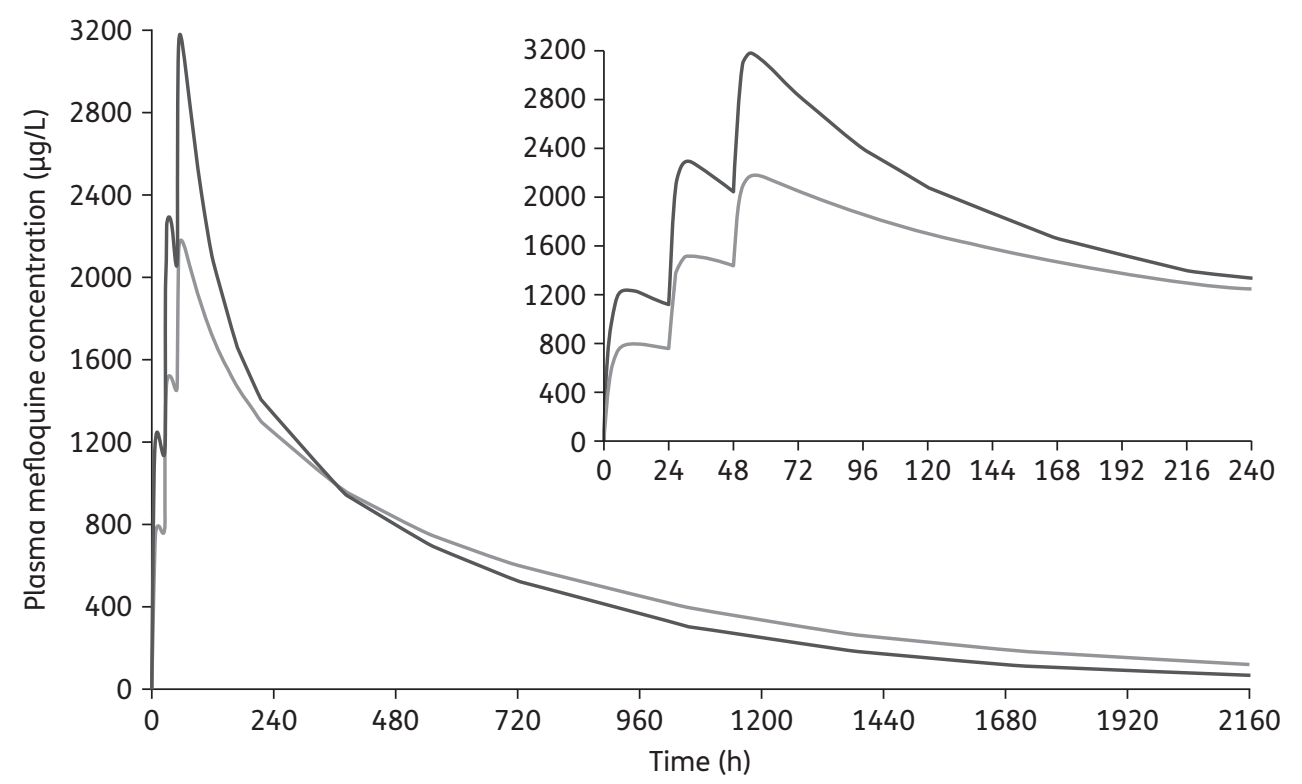

Figure 4. Simulated plasma concentration-time data for healthy volunteers ( $n=1200$; grey line) and patients with uncomplicated $P$. falciparum malaria ( $n=1075$; black line) administered $8 \mathrm{mg} / \mathrm{kg} /$ day mefloquine for 3 days.

Table 4. Post hoc pharmacokinetic parameters from simulation of 1200 healthy volunteers and 1075 patients with uncomplicated $P$. falciparum malaria administered $8 \mathrm{mg} / \mathrm{kg} /$ day mefloquine for 3 days

\begin{tabular}{lll}
\hline Parameter & Healthy volunteers & Malaria patients \\
\hline $\mathrm{CL} / \mathrm{F}(\mathrm{L} / \mathrm{h})$ & $1.16(0.586-2.02)$ & $1.14(0.583-1.91)$ \\
$V_{\mathrm{C}} / \mathrm{F}(\mathrm{L})$ & $598(294-1053)$ & $337(169-582)$ \\
$\mathrm{CL} L_{D} / \mathrm{F}(\mathrm{L} / \mathrm{h})$ & 1.34 & 1.34 \\
$V_{P} / \mathrm{F}(\mathrm{L})$ & 186 & 186 \\
$K_{\mathrm{A}}\left(\mathrm{h}^{-1}\right)$ & $1.18(0.407-2.58)$ & $1.21(0.385-2.68)$ \\
$t_{\frac{1}{2} A}(\mathrm{~h})$ & $66.8(50.8-80.0)$ & $52.8(35.1-68.7)$ \\
$t_{\frac{1}{2} \mathrm{~B}}(\mathrm{~h})$ & $566(269-1050)$ & $407(219-705)$ \\
$T_{>\text {MIC }}(\mathrm{h})$ & $893(436-1575)$ & $766(362-1362)$ \\
\hline
\end{tabular}

$\mathrm{CL} / \mathrm{F}$, clearance; $V_{C} / F$, volume of distribution of the central compartment; $\mathrm{CL}_{\mathrm{D}} / \mathrm{F}$, intercompartmental clearance; $V_{\mathrm{P}} / \mathrm{F}$, volume of distribution of the peripheral compartment; $K_{\mathrm{A}}$, absorption rate constant; $t_{\frac{1}{2} \mathrm{~A}}$, absorption half-life; $t_{\frac{1}{2} B}$, elimination half-life; $T_{>\text {MIC }}$, time above the MIC (500 $\left.\mu \mathrm{g} / \mathrm{L}\right)$.

Data are expressed as mean (5th-95th percentile interval).

clearance values than the (+)-enantiomer. ${ }^{25,26}$ Studies have also reported differences in the tissue and erythrocyte uptake of (+)and (-)-mefloquine as well as stereoselective interaction of mefloquine enantiomers with P-glycoprotein..$^{25,27-29}$ Consequently, it is possible that the observed differences in the distribution of mefloquine between malaria patients and healthy controls in this study may be explained by differences in the relative contribution of each enantiomer to the overall pharmacokinetics of mefloquine.

Malaria infection is also associated with a number of physiological changes that may affect mefloquine pharmacokinetics, in particular changes in haematocrit caused by malaria infection-induced haemolysis and/or altered haematopoiesis; in fact, haematocrit levels for patients in this study were, on average,
$7 \%$ lower than those in healthy controls (data not shown). These lower red blood cells levels would result in a reduction in the uptake of mefloquine into the erythrocyte compartment, therefore increasing the free drug concentration in plasma and reducing the volume of distribution. The adult malaria patients enrolled in this study had no or mild anaemia; the contribution of anaemia is expected to be much greater in chronically infected African children.

It is most likely that the observed changes in mefloquine pharmacokinetics in the presence of malaria infection may be attributable to a combination of some or all of these factors. However, it is important to note that this is further complicated by the time course of the malaria infection after treatment with mefloquine, i.e. after mefloquine treatment, the patient parasitic load decreases and the physiological disturbances associated with malaria infection progressively return to normal. Consequently, in these patients, the early portion of the concentration-time profile is characterized by 'patient pharmacokinetics' whereas the terminal phase would be representative of the pharmacokinetics of mefloquine in healthy volunteers. Given that the volume of distribution is dictated by the early phase of the concentration-time profile and clearance is more affected by the terminal phase, it is conceivable that the physiological mechanisms proposed above (such as changes in $\alpha_{1}$-acid glycoprotein levels and haematocrit) would result in observed changes in the volume of distribution of mefloquine, but any changes in the clearance of the drug would not be seen as the patients would have been 'healthy' by the end of the study. In fact, whilst $\alpha_{1}$-acid glycoprotein levels were not available, haematocrit values for the patients within this study progressively increase throughout the treatment period and were equivalent to those in healthy volunteers by day 28 (data not shown). Further examination of the dynamic changes in these physiological processes and their impact on the pharmacokinetics of mefloquine and other drugs throughout the time course of malaria infection is needed. 


\section{Acknowledgements}

We are grateful to the participating volunteers and patients and to the staff of the Hospital for Tropical Diseases and Universiti Sains Malaysia for their contribution to the clinical studies. We would also like to thank Dr M. Vaillant for the clinical databases.

\section{Funding}

The clinical trials were funded by the Drugs for Neglected Diseases Initiative (DNDi).

\section{Transparency declarations}

None to declare.

\section{Disclaimer}

P. L. O. is a staff member of the WHO; the author alone is responsible for the views expressed in this publication and they do not necessarily represent the decisions, policy or views of the WHO.

\section{Supplementary data}

Supplementary data are available at JAC Online (http://jac.oxfordjournals. org/).

\section{References}

1 WHO. World Malaria Report. Geneva: WHO, 2011.

2 WHO. Guidelines for the Treatment of Malaria, 2nd edn. Geneva: WHO, 2010.

3 Nosten F, van Vugt M, Price R et al. Effects of artesunate-mefloquine combination on incidence of Plasmodium falciparum malaria and mefloquine resistance in western Thailand: a prospective study. Lancet 2000; 356: 297-302.

4 Palmer KJ, Holliday SM, Brogden RN. Mefloquine. A review of its antimalarial activity, pharmacokinetic properties and therapeutic efficacy. Drugs 1993; 45: 430-75.

5 Luxemburger C, van Vugt M, Slight Tet al. Early vomiting of mefloquine in children with malaria is not modified by the timing of antipyretic treatment. Trans R Soc Trop Med Hyg 1998; 92: 562-3.

6 Simpson JA, Price R, ter Kuile F et al. Population pharmacokinetics of mefloquine in patients with acute falciparum malaria. Clin Pharmacol Ther 1999; 66: 472-84.

7 WHO. Guidelines for the Treatment of Malaria. Geneva: WHO, 2006.

8 Price R, Simpson JA, Teja-Isavatharm P et al. Pharmacokinetics of mefloquine combined with artesunate in children with acute falciparum malaria. Antimicrob Agents Chemother 1999; 43: 341-6.

9 Boudreau EF, Fleckenstein L, Pang LW et al. Mefloquine kinetics in cured and recrudescent patients with acute falciparum malaria and in healthy volunteers. Clin Pharmacol Ther 1990; 48: 399-409.

10 Karbwang J, Back DJ, Bunnag D et al. A comparison of the pharmacokinetics of mefloquine in healthy Thai volunteers and in Thai patients with falciparum malaria. Eur J Clin Pharmacol 1988; 35: 677-80.

11 Looareesuwan S, White NJ, Warrell DA et al. Studies of mefloquine bioavailability and kinetics using a stable isotope technique: a comparison of Thai patients with falciparum malaria and healthy Caucasian volunteers. Br J Clin Pharmacol 1987; 24: 37-42.
12 Olliaro P, Ramanathan S, Vaillant M et al. Pharmacokinetics and comparative bioavailability of artesunate and mefloquine administered separately or as a fixed combination product to healthy volunteers and patients with uncomplicated Plasmodium falciparum malaria. J Bioequiv Availab 2010; 2: 59-66.

13 Krudsood S, Looareesuwan S, Tangpukdee $\mathrm{N}$ et al. New fixeddose artesunate-mefloquine formulation against multidrug-resistant Plasmodium falciparum in adults: a comparative phase IIb safety and pharmacokinetic study with standard-dose nonfixed artesunate plus mefloquine. Antimicrob Agents Chemother 2010; 54: 3730-7.

14 Lai CS, Nair NK, Mansor SM et al. An analytical method with a single extraction procedure and two separate high performance liquid chromatographic systems for the determination of artesunate, dihydroartemisinin and mefloquine in human plasma for application in clinical pharmacological studies of the drug combination. J Chromatogr B 2007; 857: 308-14. 15 Savic RM, Jonker DM, Kerbusch T et al. Implementation of a transit compartment model for describing drug absorption in pharmacokinetic studies. J Pharmacokinet Pharmacodyn 2007; 34: 711-26.

16 Ashley EA, Stepniewska K, Lindegardh N et al. Population pharmacokinetic assessment of a new regimen of mefloquine used in combination treatment of uncomplicated falciparum malaria. Antimicrob Agents Chemother 2006; 50: 2281-5.

17 Simpson J, Aarons L, Price R et al. The influence of body weight on the pharmacokinetics of mefloquine. Br J Clin Pharmacol 2002; 53: 337-8.

18 Charles BG, Blomgren A, Nasveld PE et al. Population pharmacokinetics of mefloquine in military personnel for prophylaxis against malaria infection during field deployment. Eur J Clin Pharmacol 2007; 63: 271-8.

19 Svensson US, Alin H, Karlsson MO et al. Population pharmacokinetic and pharmacodynamic modelling of artemisinin and mefloquine enantiomers in patients with falciparum malaria. Eur J Clin Pharmacol 2002; 58: $339-51$.

20 Zsila F, Visy J, Mady G et al. Selective plasma protein binding of antimalarial drugs to $\alpha_{1}$-acid glycoprotein. Bioorg Med Chem 2008; 16: 3759-72.

21 Kremer JM, Wilting J, Janssen LH. Drug binding to human $\alpha$-1-acid glycoprotein in health and disease. Pharmacol Rev 1988; 40: 1-47.

22 Mackichan JJ. Influence of protein binding and use of unbound (free) drug concentrations. In: Burten ME, Shaw LM, Schentag JJ et al., eds. Pharmacokinetics and Pharmacodynamics: Principles of Therapeutic Drug Monitoring. Baltimore, MD: Lippincott Williams \& Wilkins, 2006; 82-120.

23 Voulgari F, Cummins P, Gardecki TI et al. Serum levels of acute phase and cardiac proteins after myocardial infarction, surgery, and infection. $\mathrm{Br}$ Heart J 1982; 48: 352-6.

24 Johnson JA. Influence of race or ethnicity on pharmacokinetics of drugs. J Pharm Sci 1997; 86: 1328-33.

25 Brocks DR, Mehvar R. Stereoselectivity in the pharmacodynamics and pharmacokinetics of the chiral antimalarial drugs. Clin Pharmacokinet 2003; 42: 1359-82.

26 Gimenez F, Pennie RA, Koren G et al. Stereoselective pharmacokinetics of mefloquine in healthy Caucasians after multiple doses. J Pharm Sci 1994; 83: 824-7.

27 Hellgren U, Jastrebova J, Jerling M et al. Comparison between concentrations of racemic mefloquine, its separate enantiomers and the carboxylic acid metabolite in whole blood serum and plasma. Eur J Clin Pharmacol 1996; 51: 171-3.

28 Pham YT, Nosten F, Farinotti R et al. Cerebral uptake of mefloquine enantiomers in fatal cerebral malaria. Int $J$ Clin Pharmacol Ther 1999; 37: 58-61.

29 Vidrequin S, Gimenez F, Basco LK et al. Uptake of mefloquine enantiomers into uninfected and malaria-infected erythrocytes. Drug Metab Dispos 1996; 24: 689-91. 